\title{
Chronic traumatic encephalopathy in two former Australian National Rugby League players
}

\author{
Michael E. Buckland ${ }^{1,2^{*}}$ (D) Joanne Sy ${ }^{1}$, Istvan Szentmariay ${ }^{3}$, Alexandra Kullen ${ }^{3}$, Maggie Lee ${ }^{1}$, Antony Harding ${ }^{1}$, \\ Glenda Halliday ${ }^{4}$ and Catherine M. Suter ${ }^{1,5}$
}

Keywords: Chronic traumatic encephalopathy, CTE, Rugby league, Tau, ARTAG

To the Editor:

Historically the term chronic traumatic encephalopathy (CTE) was used synonymously with 'punch-drunk' to describe the neurological deficits of ex-boxers thought due to repeated blows to the head [3]. In the twenty-first century, CTE has come to define a neuropathological diagnosis associated with repeated head injury, with the entity-defining lesion consisting of phosphorylated Tau (pTau) accumulation in neurons and astrocytes in a perivascular distribution at the depths of sulci [8]. The clinical presentation of CTE bears many features similar to the punch-drunk syndrome as originally described [2, 12]. A recent retrospective analysis of the brains in the seminal 1973 Corsellis study of punch-drunk ex-boxers boxers found that half (7 of 14) had neuropathology definitive of CTE [6].

While the above would appear to provide persuasive evidence in support of a distinct disease associated with repetitive head injury in sport, there remains a fair degree of scepticism over CTE (e.g [11]). This is due in large part to the changing semantics of the term, and to the lack of a distinct or discrete clinical syndrome. In the USA, active research into CTE in the National Football League is going some way to address the latter issue, but in other sporting nations such as Australia, awareness of the potential for CTE in contact sports is hampered by the absence of clear cases in the literature. Here we report the finding of CTE pathology in the

\footnotetext{
* Correspondence: michael.buckland@sydney.edu.au

'Department of Neuropathology, Royal Prince Alfred Hospital, 94 Mallet St, Camperdown, NSW 2050, Australia

${ }^{2}$ Discipline of Pathology, School of Medical Sciences, Brain \& Mind Centre,

University of Sydney, Camperdown, NSW 2006, Australia

Full list of author information is available at the end of the article
}

brains of two former Australian National Rugby League (NRL) players. To our knowledge these are the first reported cases of CTE in rugby league in the world, and only the second and third cases of CTE ever reported in Australian sportspeople.

Both cases were middle-aged ex-professionals who had each played more than 150 first grade NRL games over many years. Case 1 had a successful career after retirement, and had been working up until his death. He did not abuse tobacco, alcohol, or other drugs. Family members reported increasing reliance on aide-mémoires for daily activities in the years prior to death, and recent difficulties remembering details of a significant life event. Case2 had some issues during his transition to a postplaying career, but was productively employed up until his death.

Table 1 summarises the pertinent neuropathology of each case. Macroscopically, neither exhibited brain atrophy or evidence of prior traumatic brain injury. Case1 had a small cavum septum pellucidum. Microscopically, both cases had neocortical foci of the pathognomonic lesions of CTE: pTau (AT8) accumulation concentrated irregularly at the sulcal depths in a perivascular distribution. pTau was present in neurons as tangle and pretangle pathology, as well as in neurites and astrocytes. In Case1, eight definitive CTE foci were found across six of eight neocortical blocks examined (bilateral superior/ middle frontal gyri, superior/middle temporal gyri, and superior parietal lobules) (Fig. 1a, b); pTau was absent in occipital lobes (striate and peristriate cortex). In Case2, four CTE foci were found in two of four neocortical blocks examined (superior/middle frontal gyri, superior/ middle temporal gyri) (Fig. 1c, d). In non-sulcal neocortex, neurons with tangle and pretangle pathology were 
Table 1 Summary of neuropathogical features of brains of two former NRL players

\begin{tabular}{lll}
\hline & Case1 & Case2 \\
\hline Tau pathology & & Present \\
Depths of cortical sulci (neuronal \& astrocytic), perivascular & Present & Present \\
Superficial cortical layers & Present & Absent \\
CA2 (neuronal, neuritic), CA4 (proximal dendritic swellings) & Present & Present \\
Substantia nigra and raphe nuclei (neuronal, neuritic) & Present & Present \\
Subpial \& periventricular thorny astrocytes & Present & Present \\
Large grain-like \& dot-like structures & Present & \\
Other supportive pathology & & Absent \\
Dilatation of IIIrd ventricle & Absent & Absent \\
Septal abnormalities & Present & \\
Other pathology & & Absent \\
Vascular disease & Absent & Absent \\
Beta-A4 (amyloid) & Present (Thal phase 1) & 0 \\
CERAD score & 0 & Present (amygdala, hippocampus, entorhinal cortex, \\
pTDP-43 & inferior temporal cortex) & Absent \\
Alpha-synuclein & Absent & Absent \\
ARTAG & moderate (medial temporal) \\
Diagnosis & Stage 3 CTE & mild (medial temporal) \\
\hline
\end{tabular}

present mostly in cortical layers II and III (moderate frequency in Case2, low in Case1).

Case1 also demonstrated medial temporal lobe involvement, with neuronal loss in the hippocampal CA1 region, subiculum, and amygdala, with prominent ghost tangle formation in amygdala. pTau staining was widespread, with notable pretangle neuronal staining in CA4 and CA2 regions, with prominent staining of proximal dendritic segments in CA4 (Fig. 1e). Both cases had some Tau-positive globose neurofibrillary tangles (GFTs; Fig. 1f) and neurites in the substantia nigra, accompanied by mild neuronal loss, gliosis, and pigment incontinence. Case1 also showed involvement of raphe nuclei, locus coeruleus and dorsal motor nucleus of vagus.
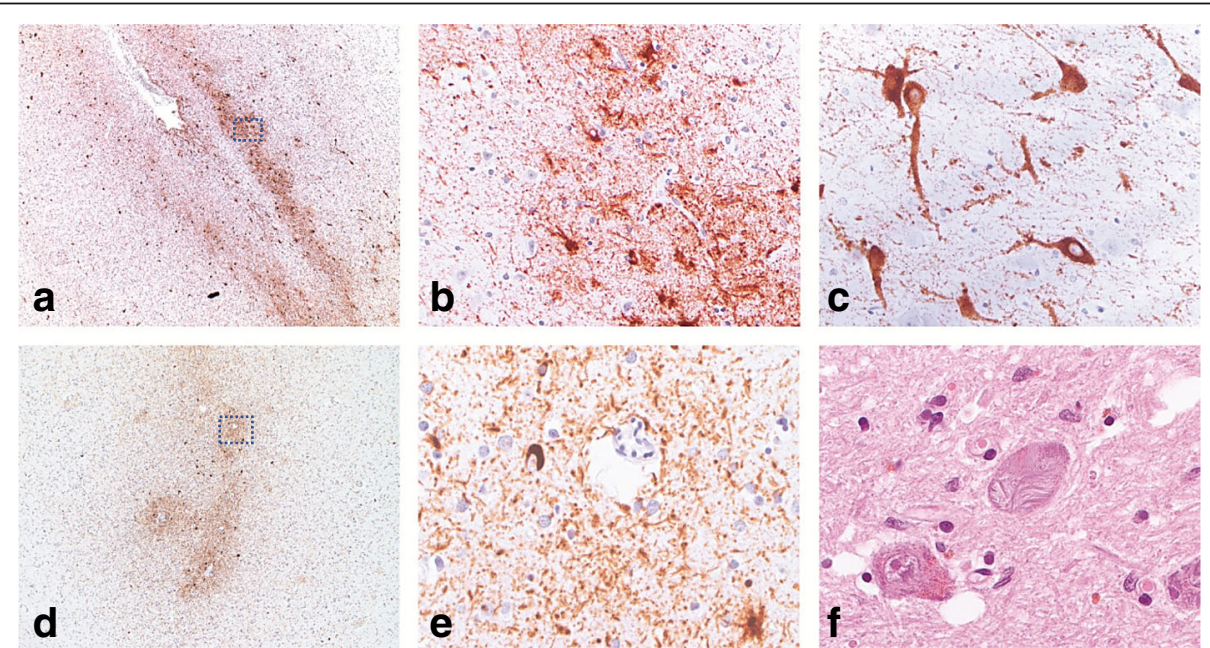

Fig. 1 Phosphorylated Tau immunohistochemistry (a-e) and haematoxylin and eosin staining (f) in Case1 (a-c) and Case2 (d-f). (a) pTAu staining is irregularly distributed th the sulcal depths in both neurons and glia. (b) high power view of boxed area in (a) showing perivascular accentuation of pTau staining. (c) prominent proximal dendrite staining with pTau in CA4 neurons in Case1. (d) perivascular pTAu staining in sulcal depths of Case2 (frontal cortex). (e) high power view of boxed area in (d). (f) globose fibrillary tangles in the substantia nigra with some pigment incontinence and gliosis in Case2 
Thorny astrocytic Tau staining in subpial and subependymal locations in medial temporal lobe was also seen in both cases, indicating co-existent aging-related tau astrogliopathy (ARTAG) [7].

In both cases, the neuronal inclusions comprised both $3 \mathrm{R}$ and $4 \mathrm{R}$ Tau isoforms, with $4 \mathrm{R}$ Tau preferentially labelling neuritic and glial pathology. In the ghost tangles of Case 1, 3R Tau was the dominant isoform. Case 1 also contained medial temporal lobe TDP-43 pathology, and low numbers of diffuse plaques in frontal and temporal neocortex. Coexistent pathology is detailed in Table 1 . The distinctive Tau pathology seen in both cases is diagnostic of CTE; the medial temporal lobe involvement indicates that Case1 corresponds to stage III pathology, while Case2 best corresponds to Stage II pathology [9].

Rugby league is a very popular amateur and professional sport in Australia with a player base of around half a million people https://www.clearinghouseforsport.gov.au/. It is distinct from rugby union ('rugby'), having established itself as a separate sporting code over 100 years ago. It is also popular in Pacific Island nations, New Zealand, the United Kingdom and France. There is frequent high force tackling of players, and head impact during tackling is common. The rate of concussions in professional rugby league is estimated at 8.92 per 1000 match hours (i.e. one concussion every 3.35 games [4]), and may be significantly higher in youth rugby league [5]. Currently, repeated mild traumatic brain injury, both concussive and subconcussive, is the only known likely risk factor for CTE [1]. There is little available data on long-term neurological outcomes in rugby league players, however a recent assessment of 25 retired NRL players identified significant motor and cognitive changes, along with neurophysiological alterations compared with matched controls with no history of contact sports [10].

While this report is limited by the paucity of antemortem clinical information, the finding of two clear CTE diagnoses in our department is nonetheless remarkable. RPA Neuropathology is the only neuropathology department in the most populous state in Australia. Over the last four years, the routine service has examined 470 brain autopsy specimens, with 50 undergoing neocortical Tau immunostaining for various indications. These cases we present here are the only two cases of CTE we have identified in routine practice, and it is noteworthy that they are both in elite rugby league players.

Given that brain autopsy is rarely pursued these days, even in cases of suicide referred to the Coroner, it is difficult to assess whether these two CTE cases are serendipitous findings, or emblematic of a more common issue with Rugby League and other Australian football codes. Our findings underscore the urgent need for further studies into CTE in sportspeople and other at-risk populations internationally, in order to define and act upon the occupational and public health implications of this disease.

\section{Abbreviations}

ARTAG: Aging-related tau astrogliopathy; CTE: Chronic traumatic encephalopathy; NRL: National rugby league

\section{Acknowledgements}

We thank Dianna Van Beek and Karina Hammond for expert technical assistance.

\section{Authors' contributions}

MEB, JS, GH performed neuropathological analysis. MEB and CMS drafted the manuscript. MEB, ML, IS, AK contributed to acquisition and/or analysis of data. All authors were involved in revisions of the manuscript. All authors read and approved the final manuscript.

\section{Funding}

Nil

\section{Availability of data and materials}

Data sharing is not applicable to this article (no datasets were generated or analysed during the current study).

Ethics approval and consent to participate

Ethics approval for publication has been waived.

Consent for publication

Not required.

\section{Competing interests}

The authors declare that they have no competing interests.

\section{Author details}

'Department of Neuropathology, Royal Prince Alfred Hospital, 94 Mallet St, Camperdown, NSW 2050, Australia. ${ }^{2}$ Discipline of Pathology, School of Medical Sciences, Brain \& Mind Centre, University of Sydney, Camperdown, NSW 2006, Australia. ${ }^{3}$ Forensic and Analytical Science Service, Lidcombe, NSW 2141, Australia. ${ }^{4}$ Central Clinical School, Brain \& Mind Centre, University of Sydney, Camperdown, NSW 2006, Australia. ${ }^{5}$ Faculty of Medicine, University of New South Wales, Kensington, NSW 2052, Australia.

Received: 13 June 2019 Accepted: 13 June 2019

Published online: 27 June 2019

\section{References}

1. CDC (2019) Answering questions about chronic traumatic encephalopathy (CTE) https://www.cdc.gov/traumaticbraininjury/pdf/ CDC-CTE-FactSheet-508.pdf

2. Corsellis JA, Bruton CJ, Freeman-Browne D (1973) The aftermath of boxing. Psychol Med 3:270-303

3. Critchley M (1957) Medical aspects of boxing, particularly from a neurological standpoint. Br Med J 1:357-362

4. Gardner AJ, Howell DR, Levi CR, Iverson GL (2017) Evidence of concussion signs in National Rugby League Match Play: a video review and validation study. Sports Med Open 3:29. https://doi.org/10.1186/s40798-017-0097-9

5. Gardner AJ, Kohler RM, Levi CR, Iverson GL (2017) Usefulness of video review of possible concussions in National Youth Rugby League. Int J Sports Med 38:71-75. https://doi.org/10.1055/s-0042-116072

6. Goldfinger MH, Ling H, Tilley BS, Liu AKL, Davey K, Holton JL, Revesz T, Gentleman SM (2018) The aftermath of boxing revisited: identifying chronic traumatic encephalopathy pathology in the original Corsellis boxer series. Acta Neuropathol 136:973-974. https://doi.org/10.1007/s00401-018-1926-8

7. Kovacs GG, Ferrer I, Grinberg LT, Alafuzoff I, Attems J, Budka H, Cairns NJ, Crary JF, Duyckaerts C, Ghetti B et al (2016) Aging-related tau astrogliopathy (ARTAG): harmonized evaluation strategy. Acta Neuropathol 131:87-102. https://doi.org/10.1007/s00401-015-1509-x

8. McKee AC, Cairns NJ, Dickson DW, Folkerth RD, Keene CD, Litvan I, Perl DP, Stein TD, Vonsattel JP, Stewart W et al (2016) The first NINDS/NIBIB consensus meeting to define neuropathological criteria for the diagnosis of chronic traumatic encephalopathy. Acta Neuropathol 131:75-86. https://doi. org/10.1007/s00401-015-1515-z

9. McKee AC, Stern RA, Nowinski CJ, Stein TD, Alvarez VE, Daneshvar DH, Lee HS, Wojtowicz SM, Hall G, Baugh CM et al (2013) The spectrum of disease in 
chronic traumatic encephalopathy. Brain 136:43-64. https://doi.org/10.1093/ brain/aws307

10. Pearce AJ, Rist B, Fraser CL, Cohen A, Maller JJ (2018) Neurophysiological and cognitive impairment following repeated sports concussion injuries in retired professional rugby league players. Brain Inj 32:498-505. https://doi. org/10.1080/02699052.2018.1430376

11. Randolph C (2018) Chronic traumatic encephalopathy is not a real disease. Arch Clin Neuropsychol 33:644-648. https://doi.org/10.1093/arclin/acy063

12. Stern RA, Daneshvar DH, Baugh CM, Seichepine DR, Montenigro PH, Riley DO, Fritts NG, Stamm JM, Robbins CA, McHale L et al (2013) Clinical presentation of chronic traumatic encephalopathy. Neurology 81:11221129. https://doi.org/10.1212/WNL.0b013e3182a55f7f

\section{Publisher's Note}

Springer Nature remains neutral with regard to jurisdictional claims in published maps and institutional affiliations.

Ready to submit your research? Choose BMC and benefit from:

- fast, convenient online submission

- thorough peer review by experienced researchers in your field

- rapid publication on acceptance

- support for research data, including large and complex data types

- gold Open Access which fosters wider collaboration and increased citations

- maximum visibility for your research: over $100 \mathrm{M}$ website views per year

At BMC, research is always in progress.

Learn more biomedcentral.com/submissions 